Jill T. Jesurum

Cindy J. Fuller

Carles A. Velez

Merrill P. Spencer $\dagger$

Kimberly A. Krabill

William H. Likosky

William A. Gray

John V. Olsen

Mark Reisman

\section{Migraineurs with patent foramen ovale have larger right-to-left shunt despite similar atrial septal characteristics}

Received: 19 March 2007

Accepted in revised form: 25 July 2007

Published online: 24 September 2007

J.T. Jesurum • C.J. Fuller • C.A. Velez • M.P. Spencer • K.A. Krabill • W.H. Likosky •

W.A. Gray • J.V. Olsen • M. Reisman Departments of Cardiovascular Research and Neurology,

Swedish Medical Center.

Seattle, WA, USA

J. Jesurum (两)

Swedish Heart \& Vascular Institute,

Swedish Medical Center,

801 Broadway, Suite 927,

Seattle, WA 98122, USA

Tel.: +1-206-386-6290

Fax: +1-206-386-6073

e-mail: jill.jesurum@swedish.org

$\dagger$ Deceased

\begin{abstract}
The objective of the study was to assess differences in proportion of large right-to-left shunt (RLS) and atrial septal characteristics between migraineurs and non-migraineurs referred for transcatheter closure of patent foramen ovale (PF0). This retrospective study took place in a large metropolitan medical centre. The patients were migraineurs with aura $(n=52)$, migraineurs without aura $(n=19)$ and non-migraineurs $(\mathrm{n}=149)$. RLS was evaluated before closure using bilateral power m-mode transcranial Doppler at rest and after calibrated, sustained Valsalva manoeuvre, and graded with a validated $0-5$ scale. Intracardiac echocardiography was used to assess atrial septal characteristics. Migraineurs had a
\end{abstract}

higher proportion of large RLS (Grade IV or V) than nonmigraineurs at rest and after calibrated Valsalva (rest, $p=0.04$; Valsalva, $p=0.01$ ). Atrial septal characteristics were similar between groups. Migraine is associated with larger RLS at rest and strain; however migraine status does not predict PFO characteristics.

Keywords Migraine $\cdot$ Patent foramen ovale $\cdot$ Transcranial Doppler

\section{Introduction}

Migraine headaches are associated with high morbidity and significant loss of productivity $[1,2]$. Across all age groups, migraineurs are more likely to experience ischaemic stroke than non-migraineurs (relative risk [RR] 2.16, 95\% confidence interval $[\mathrm{CI}]$ 1.89-2.48), especially migraineurs who have aura (RR 2.88, 95\% CI 1.89-4.39) [3]. Epidemiological data indicate an increased incidence of patent foramen ovale (PFO) with intermittent right-to-left interatrial shunting (RLS) in migraineurs and cryptogenic ischaemic stroke patients compared to the general population [4-6]. The incidence of RLS in migraineurs in one study was $41 \%$, relative to $16 \%$ in healthy controls [4]. This observation has led researchers to speculate that cellular and/or humoral factors in the venous system bypass pulmonary filtration through a PFO and trigger migraine symptoms in susceptible individu- 
als $[7,8]$. RLS may repeatedly expose hyperexcitable occipital neurons to microemboli or chemicals, setting up cortical spreading depression. The resulting ischaemia or increased local levels of serotonin may stimulate trigeminal nerve endings, resulting in headache pain [9]. Although controversial, preliminary support for this theory has come from several non-randomised studies showing that transcatheter PFO closure resulted in either complete resolution or a significant $(>50 \%)$ reduction in migraine symptoms [10-12]. Despite these findings, the importance of PFO in migraineurs continues to be debated because PFO is present in $20 \%-30 \%$ of the general population and in a large proportion of patients without vascular disorders [13].

Characteristics of the interatrial septum in patients with PFO, particularly coexisting atrial septal aneurysm (ASA), have been implicated in increased risk of stroke [14-16] and in migraine with aura [17]. In a case-control study using transoesophageal echocardiography (TEE) [18], a PFO measuring $>4 \mathrm{~mm}$ in diameter was associated with higher risk for stroke or transient ischaemic attack (TIA).

Researchers have speculated that a large RLS may be one potential reason for the high risk of ischaemic stroke in migraineurs with PFO [19-21]. Migraineurs with aura and history of stroke were more likely to have a clinically relevant RLS ( $>6$ microbubbles visualised in the left atrium during contrast transthoracic echocardiography) than population controls $(84 \%$ vs. $12 \%, p<0.001)$ [6]. Schwerzmann et al. [19] reported that the presence of a moderate or large RLS, as assessed by TEE, increased the odds of migraine with aura 7.78-fold (95\% CI 2.53-29.3, $p<0.001)$. Transcranial Doppler assessments have also shown that migraineurs have a larger degree of RLS than non-migraineurs [20, 21].

To date, published studies documenting the degree of RLS in migraineurs have failed to describe the atrial septal characteristics; therefore, the purpose of this study was to assess the degree of RLS with power m-mode transcranial Doppler (pm-TCD) and to report atrial septal characteristics in migraineurs and non-migraineurs ( $\mathrm{MH}-$ ) referred for transcatheter PFO closure. We postulated that in comparison to $\mathrm{MH}-$, migraineurs would have a higher proportion of large RLS and large PFO diameter, which may result in a greater susceptibility to vascular events such as migraine and stroke.

\section{Methods}

Design

The retrospective, observational analysis was based on a series of 237 consecutive patients who underwent transcatheter PFO closure for prevention of recurrent stroke and/or TIA between April 2001 and June 2005 at the Swedish Medical Center in Seattle,
Washington. Approval was obtained from the Western Institutional Review Board (Olympia, WA) to conduct retrospective analysis of clinical data with a waiver of written informed consent. This study was performed in accordance with the ethical standards of the 1964 Declaration of Helsinki. Eligibility criteria for transcatheter PFO closure included a presumed paradoxical embolic event, evidence of provocable RLS confirmed by contrast-enhanced, bilateral pmTCD and/or TEE, and probe patency of the septal tunnel during catheterisation. Patients with coexisting atrial septal defects were excluded from the final analysis.

All patients underwent neurological evaluation prior to $\mathrm{PFO}$ closure as part of the routine standard of clinical care. Ischaemic stroke was defined as an acute focal neurological event with corresponding positive findings on magnetic resonance imaging, regardless of duration of clinical symptoms; TIA was defined as a temporary, reversible focal neurological event without changes on magnetic resonance imaging. Information was collected using a patient questionnaire that assessed cardiac and neurological symptoms, as well as standardised migraine information. Migraine data were extracted from patient questionnaires and retrospectively analysed. Patients were stratified into migraineur and $\mathrm{MH}-$ groups by a neurologist according to the International Headache Society diagnostic criteria for migraine headache [22]. Migraineurs were further classified by the presence (MA+) or absence (MA-) of aura.

All patients underwent baseline pm-TCD examination of both middle and anterior cerebral arteries utilising a 100M digital 2MHz Doppler platform (Spencer Technologies, Seattle, WA). Using a standardised technique [23], agitated saline was injected into an antecubital vein during normal respiration and immediately following a calibrated $(40 \mathrm{mmHg})$, sustained (10 s) Valsalva manoeuvre. Appearance of microbubbles in the middle and anterior cerebral arteries was monitored for 1 min using bilateral pm-TCD. Primary clinical endpoints included the degree of RLS, as assessed by the number of microbubbles detected during normal respiration and immediately following Valsalva. Spontaneous or provoked RLS was semi-quantitatively graded using a previously validated classification system (Grade 0=0, Grade $\mathrm{I}=1-10$, Grade $\mathrm{II}=11-30$, Grade III=31-100, Grade VI=101-300, Grade V>300) [23]. When microbubbles were too numerous to count (shower or curtain effect), the result was recorded as 301 or Grade V.

Intracardiac echocardiography (ICE) was performed during the PFO closure procedure utilising an ACUSON AcuNav system (Siemens, San Diego, CA) to evaluate atrial septal characteristics, including PFO tunnel length, balloon-stretch diameter, and presence of coexisting ASA and atrial septal defect [24]. A compliant, conforming sizing balloon was used to assess the diameter of the defect prior to device implantation. Balloon inflation was completed when the PFO tunnel was completely occluded and a small waist was visible on ICE. The inflated balloon waist diameter was measured with the aid of calibrations on the sizing balloon. The length of the septal tunnel was measured as the distance between right and left atria without balloon inflation. Atrial septal characteristics were evaluated post-procedurally by a single paediatric cardiologist (K.A.K.) who 
has an expertise in echocardiography, including transoesophageal and intracardiac techniques, and who was blinded to patient migraine status. ASA was defined as the presence of a localised protrusion of the fossa ovalis, with a base width $>15 \mathrm{~mm}$ and mobile septal excursion into the left or right atrium $>10 \mathrm{~mm}$ [25]. Atrial septal defect was defined as a predominant left-to-right interatrial shunt at rest, based on colour-flow Doppler interrogation, and echocardiographic dropout indicative of absence of a portion of the septum primum.

\section{Statistical analysis}

The primary endpoint of this study was to compare the proportion of large RLS (Grades IV-V) at rest and after calibrated Valsalva between migraineurs and non-migraineurs. The secondary endpoint was to compare the atrial septal characteristics of migraineurs and MH-. Subanalyses for primary and secondary endpoints were performed between MA+ and MA-.

Descriptive statistics were used to evaluate demographic and comorbidity data, atrial septal characteristics and RLS measurements. Continuous variables were reported using the mean, range, standard deviation and 95\% CI; comparisons were made using analysis of variance. Nominal and categorical data were reported as frequencies and percentages, and were compared using chi-square analysis and Fisher's exact tests for independent samples. Normality was assessed by examining stem-and-leaf plots and histograms, and statistically evaluated using the Kolmogorov-Smirnov test. Independent sample comparisons of RLS measurements and atrial septal characteristics were performed using the non-parametric Kruskal-Wallis $\chi^{2}$ and Mann-Whitney $U$-tests. Multivariate regression was performed to determine the effect of age and gender on RLS grade. Logistic regression was performed to assess the effects of atrial septal characteristics on large RLS at strain. Spearman's rank correlation coefficients were calculated to assess the relation between migraine frequency, atrial septal characteristics and degree of RLS at rest or strain. Power analysis was performed using noncentral $\chi^{2}$. The level of significance for the omnibus test for the primary endpoint was set at $p=0.05$. A Bonferroni correction was used to control for post hoc analyses to evaluate the proportion of large RLS between groups; therefore, the level of significance was set at $p=0.025$ (two-tailed). The threshold for statistical significance for other parameters was set at $p=0.05$. Statistical analyses were performed using the SPSS ${ }^{\mathrm{TM}}$ Advanced Models software (version 15.0.1, SPSS, Inc., Chicago, IL) and Sample Power 2.0 (SPSS).

\section{Results}

Patient population

Between April 2001 and June 2005, 237 consecutive patients (mean age, 53 \pm 15 years, 51\% women) underwent tran- scatheter PFO closure. Indications for PFO closure included prevention of recurrent cryptogenic ischaemic stroke or TIA, as evidenced by clinical and radiological findings (i.e., computerised tomography, magnetic resonance imaging). Twelve (5\%) patients who did not have pm-TCD evaluation prior to PFO closure were excluded from the final analysis and 5 ( $2 \%$ ) patients were excluded due to coexisting atrial septal defect; therefore, the final sample consisted of 220 patients. With a sample size of 220 , the study yielded a power of $74.3 \%$ to test the null hypothesis that $\mathrm{MH}+$ had a significantly greater proportion of large RLS than $\mathrm{MH}-$. All patients had adequate temporal bone windows for pm-TCD insonation.

Patients were classified by a neurologist into MA+ $(n=52,24 \%), M A-(n=19,9 \%)$ and $M H-$ groups $(n=149$, $68 \%$ ) according to migraine diagnostic criteria published by the International Headache Society [22]. Migraineurs with aura, on average, experienced 5.1 (95\% CI 3-7) migraine headaches each month of duration $>4 \mathrm{~h}$ with a mean pain intensity rating of $7.3 \pm 2.5$ on a 10 -point scale. Migraineurs without aura experienced 5.5 (95\% CI 2-9; $p=0.07$ vs. MA+) migraines per month of duration $>4 \mathrm{~h}$ with mean pain intensity of $7.6 \pm 2.4$ ( $p=0.6 \mathrm{vs}$. MA+). The average age at migraine onset was 22 \pm 10 years for MA+ and 26 \pm 16 years for MA$(p=0.2)$.

Baseline characteristics and comorbidities are presented in Table 1. Migraineurs were more likely to be female and younger than MH-. At the time of PFO closure, $90 \%$ of $\mathrm{MA}+$ and $100 \%$ of MA- patients were less than or equal to 65 years of age compared to $66 \%$ of $\mathrm{MH}-(p<0.01)$. Among all groups, there was a high incidence of pre-existing hypertension, hyperlipidaemia, hypercoagulable disorders and coronary artery disease, although few patients had pulmonary hypertension or chronic lung disease. Prior to PFO closure, 119 (54\%) patients experienced one or more clinically and radiographically confirmed strokes without TIA, $71(32 \%)$ experienced one or more TIAs without stroke and $30(14 \%)$ experienced both stroke and TIA. The proportion of patients experiencing recurrent neurological events prior to $\mathrm{PFO}$ closure was similar in all groups $(\mathrm{MA}+, 25 \%$; MA-, $37 \%$; $\mathrm{MH}-, 26 \%, p=0.6)$. At the time of initial consultation, most $(95 \%)$ patients were on anticoagulation and/or antiplatelet therapy for secondary stroke prevention.

Interatrial right-to-left shunt

At rest, migraineurs had a greater proportion of large RLS (Grades IV-V) at rest than non-migraineurs $\left(\chi^{2}=4.3, p=0.03\right)$ (Fig. 1). The degree of RLS at rest was significantly different between the three groups $\left(\chi^{2} 2=10.2, p<0.01\right)$ (Fig. 2A). Post hoc analysis revealed that MA- had a greater degree of RLS than MA+ $(Z=-2.3, p=0.02)$ and $\mathrm{MH}-(Z=-3.0$, 
$p<0.01)$. The difference in degree of RLS between MA+ and MH- was not significant $(Z=-1.3, p=0.2)$.

Following calibrated Valsalva, migraineurs had a greater proportion of large RLS than non-migraineurs $\left(\chi^{2}=5.9\right.$, $p=0.01$ ) (Fig. 1). The degree of RLS was significantly different between the three groups $\left(\chi^{2}=6.9, p=0.03\right.$ ) (Fig. 2B). Post hoc analysis revealed that MA+ had a greater degree of RLS than $\mathrm{MH}-(Z=-2.6, p<0.01)$, but not compared to MA$(Z=-1.2, p=0.2)$. The difference in the degree of RLS between MA- and MH- was not significant $(Z=-0.7, p=0.5)$. There was no significant group differences in the average change (delta) in embolic tracks between normal respiration and following calibrated Valsalva (MA+ 135 $\pm 120,95 \% \mathrm{CI}$,

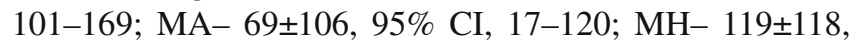
95\% CI, $100-138, \chi^{2}=4.9, p=0.09$ ) (Fig. 3). The number of embolic tracks measured at rest was significantly correlated with the number of embolic tracks measured at strain $(\mathrm{r}=0.43, p<0.01)$. In migraineurs, the average number of headaches per month was not significantly correlated with embolic tracks at rest $(\mathrm{r}=-0.02, p=0.9)$ or at strain $(\mathrm{r}=-0.07$, $p=0.6$ ). Multivariate regression revealed no significant effect of gender $(\mathrm{r}=0.08, p=0.3)$ or age at time of PFO closure $(\mathrm{r}=-0.06, p=0.4)$ on RLS grade at strain.

\section{Atrial septal characteristics}

Atrial septal characteristics, assessed by ICE, are shown in Table 2. Eighteen (35\%) MA+, 8 (42\%) MA- and 49 (33\%) MH- had coexisting ASA $(p=0.7)$. Mean ASA excursion $(p=0.2)$ and pouch width $(p=0.5)$ were not different between the three groups. Mean PFO septal tunnel length $(p=0.06)$ and PFO balloon diameter $(p=0.3$ ) were also similar between groups. Logistic regression revealed no significant effect of PFO balloon diameter $(\mathrm{r}=-0.06, p=0.4)$ or PFO tunnel length $(\mathrm{r}=-0.02, p=0.8)$ on the presence of large RLS at strain; however, the change in RLS (embolic tracks) from rest to strain was significantly correlated with PFO balloon waist diameter $(\mathrm{r}=-0.197, p=0.005)$ and PFO tunnel length $(\mathrm{r}=-0.194$, $p=0.007)$.

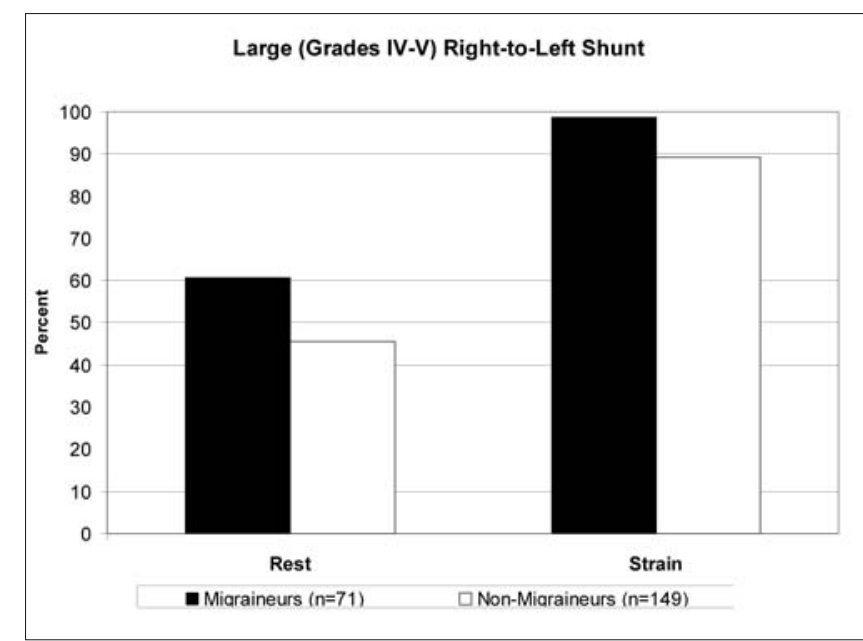

Fig. 1 Percent of migraineurs and non-migraineurs who had large (Grades IV-V) RLS at rest and following calibrated Valsalva (strain). At rest, migraineurs had a higher proportion of large RLS than non-migraineurs $\left(\chi^{2}=4.3, p=0.03\right)$ and at strain $\left(\chi^{2}=5.9, p=0.01\right)$

Table 1 Characteristics and comorbidities of migraineurs and non-migraineurs referred for transcatheter PFO closure after stroke or TIA

\begin{tabular}{|c|c|c|c|c|}
\hline & $\begin{array}{c}\text { Migraineurs } \\
\text { with aura }(n=52)\end{array}$ & $\begin{array}{c}\text { Migraineurs } \\
\text { without aura }(n=19)\end{array}$ & $\begin{array}{c}\text { Non-migraineurs } \\
(\mathrm{n}=149)\end{array}$ & $p$ value $^{\mathrm{a}}$ \\
\hline Female & $40(77)$ & $14(74)$ & $60(40)$ & $<0.001$ \\
\hline Age (years) & $47 \pm 12$ & $44 \pm 9$ & $56 \pm 16$ & $<0.001$ \\
\hline Caucasian & $49(94)$ & $18(95)$ & $136(91)$ & $\mathrm{NS}^{\mathrm{b}}$ \\
\hline Recurrent neurological events pre-closure & $13(25)$ & $7(37)$ & $38(26)$ & NS \\
\hline Coronary artery disease & $10(19)$ & $2(10)$ & $31(21)$ & NS \\
\hline Peripheral arterial disease & $4(8)$ & $1(5)$ & $9(6)$ & NS \\
\hline Diabetes mellitus & $5(10)$ & $2(10)$ & $15(10)$ & NS \\
\hline Hyperlipidaemia & $15(29)$ & $5(26)$ & $63(42)$ & NS \\
\hline Hypertension & $23(44)$ & $6(32)$ & $67(45)$ & NS \\
\hline Current or former smoker & $18(35)$ & $6(16)$ & $59(40)$ & NS \\
\hline Pulmonary hypertension & $2(4)$ & $1(5)$ & $8(5)$ & NS \\
\hline Chronic lung disease & $1(2)$ & $0(0)$ & $4(3)$ & NS \\
\hline Hypercoagulable disorder & $17(33)$ & $5(33)$ & $54(36)$ & NS \\
\hline Anticoagulation therapy & $23(44)$ & $9(47)$ & $82(55)$ & NS \\
\hline Daily antiplatelet therapy & $35(67)$ & $6(32)$ & $93(62)$ & NS \\
\hline
\end{tabular}

Data are presented as mean \pm standard deviation or frequency $(\%)$ ${ }^{\mathrm{a}} \chi^{2}$, Fisher's exact test or analysis of variance; ${ }^{\mathrm{b}} \mathrm{NS}$, not significant 


\section{Discussion}

This retrospective analysis showed that cryptogenic stroke patients with PFO and a history of migraine headaches had a higher proportion of large RLS at rest and after calibrated Valsalva than non-migraineurs despite similar atrial septal characteristics. In addition, no significant correlation was seen between PFO balloon diameter and degree of RLS. Although intracardiac haemodynamic measurements were not obtained at the time of PFO closure to rule out elevated right atrial pressure, there were no significant group differences in the percentage of patients who had a history of pulmonary hyperten- sion, chronic lung disease, heart failure or other comorbidity that could have influenced the magnitude of RLS during pmTCD evaluation.

Our patient sample was similar to other studies of PFO closure in migraineurs in the large number of women and the relatively young age at closure to prevent recurrent stroke [11, 26]. Patients with coexisting atrial septal defects were excluded from the analysis. The degree of RLS in migraineurs was not correlated with migraine frequency, consistent with the findings of a small study $(n=30)$ [27].

The finding of similar atrial septal characteristics between migraineurs and non-migraineurs was unexpected. In addition,

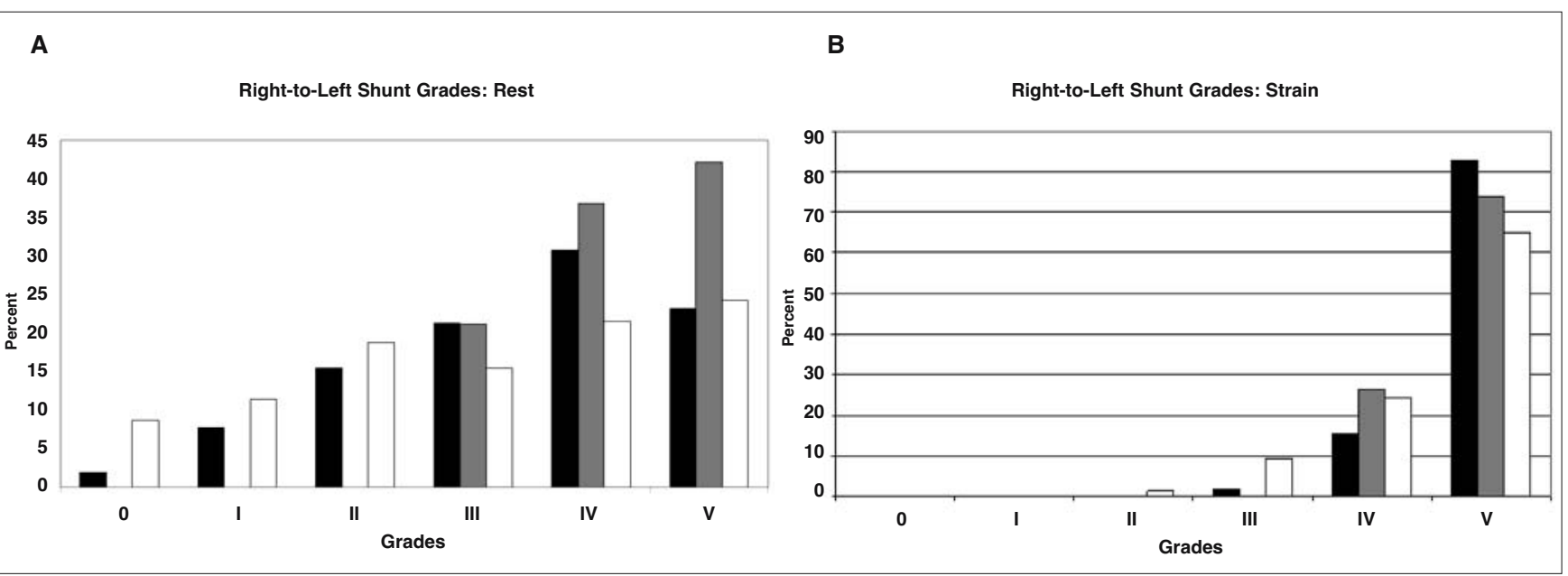

Fig. 2 Grade of RLS during normal respiration (rest, A) and after calibrated, sustained Valsalva (strain, B) in migraineurs with aura (MA+, black), migraineurs without aura (MA-, gray) and non-migraineurs ( $\mathrm{MH}-$, white). Omnibus comparisons at rest and strain were significantly different between groups (Kruskal-Wallis test: rest $\chi^{2}=10.2, p<0.01$; strain $\chi^{2}=7.2, p=0.03$ ). At rest, MA- patients had significantly higher degree of RLS than MA+ (Mann-Whitney $U$-test: $Z=-2.3, p=0.02)$ and $\mathrm{MH}-(Z=-3.0, p<0.01)$. At strain, MA+ had a greater degree of RLS than $\mathrm{MH}-(Z=-2.6, p<0.01)$

Table 2 Atrial septal characteristics and presence of large RLS in migraineurs with aura and non-migraineurs undergoing transcatheter closure of PFO

\begin{tabular}{|c|c|c|c|c|}
\hline Atrial septal characteristic & $\begin{array}{c}\text { Migraineurs } \\
\text { with aura }(n=52)\end{array}$ & $\begin{array}{c}\text { Migraineurs } \\
\text { without aura }(n=19)\end{array}$ & $\begin{array}{c}\text { Non-migraineurs } \\
\quad(n=149)\end{array}$ & $p$ value $^{\mathrm{a}}$ \\
\hline
\end{tabular}

Coexisting ASA ${ }^{\mathrm{b}}$

ASA base pouch width $(\mathrm{mm})$

ASA septal excursion (mm)

Septal tunnel length $(\mathrm{mm})$

Balloon stretch diameter ( $\mathrm{mm}$ )

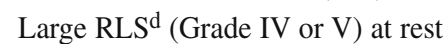

Large RLS (Grade IV or V) after calibrated,

sustained Valsalva

$\begin{array}{rr}18(35) & 8(42) \\ 21 \pm 6 & 19 \pm 3 \\ 15 \pm 4 & 13 \pm 2 \\ 11 \pm 3 & 10 \pm 3 \\ 13 \pm 3 & 12 \pm 3 \\ 28(54) & 15(79) \\ 51(98) & 19(100)\end{array}$

$19 \pm 3$

$13 \pm 2$

$10 \pm 3$

$12 \pm 3$

$19(100)$

$\begin{array}{rr}49(33) & \mathrm{NS}^{\mathrm{c}} \\ 20 \pm 5 & \mathrm{NS} \\ 16 \pm 5 & \mathrm{NS} \\ 12 \pm 3 & \mathrm{NS} \\ 13 \pm 4 & \mathrm{NS} \\ 68(46) & 0.02 \\ 133(89) & \mathrm{NS}\end{array}$

Data are presented as mean \pm standard deviation or frequency $(\%)$

apearson $\chi^{2}$, Fisher's exact test, analysis of variance or Kruskal-Wallis test

bASA, atrial septal aneurysm

cNS, non-significant

${ }^{\mathrm{d}} \mathrm{RLS}$, right-to-left interatrial shunt at baseline 
we found no significant relation between balloon diameter or septal tunnel length and large RLS. The change in RLS between rest and Valsalva was significantly correlated with balloon diameter and septal tunnel length, although the coefficients were small ( $r=-0.197$ and $r=-0.194$, respectively). The present study is the first to report direct measurements of atrial septal characteristics by ICE in migraineurs. The balloonsizing technique utilised compared favourably with TEE sizing of PFO in one study [28]. ASA and PFO were more common in migraineurs than non-migraineurs in one cohort (OR 2.71; 95\% CI 1.36-5.41, $p=0.005$ ) [29]. Carerj et al. [17] reported that MA+ patients had a higher prevalence of ASA (10 of 35, 28.5\%) than migraineurs without aura (2 of 55, $3.6 \%, p<0.005$ ) or controls ( 1 of $53,1.9 \%, p<0.005)$. Thirty percent ( 3 of 10) of the ASA-positive MA+ patients studied by these investigators had coexisting PFO, whereas $100 \%$ of migraineurs without aura and control patients with ASA had coexisting PFO [17].

In our patient cohort, there was no relation between large RLS and stroke or TIA. In a secondary analysis, we determined that the percentages of $\mathrm{MA}+, \mathrm{MA}-$ and $\mathrm{MH}-$ in those with a history of stroke (without TIA) were not different (MA+ 69\%, MA- 68\%, MH- 67\%, $p=1.0$ ). Furthermore, there were no differences in the proportion of large RLS between patients who had a history of either stroke or TIA (stroke 49\%, TIA 54\%; $\chi^{2}=0.4, p=0.5$ ).

The relation between atrial septal characteristics and recurrent stroke has been explored. Schuchlenz et al. [18] found that PFO diameter $>4 \mathrm{~mm}$ was associated with a significantly greater risk of TIA, stroke and recurrent stroke. Diameter was assessed as the maximum separation between the septum pri-

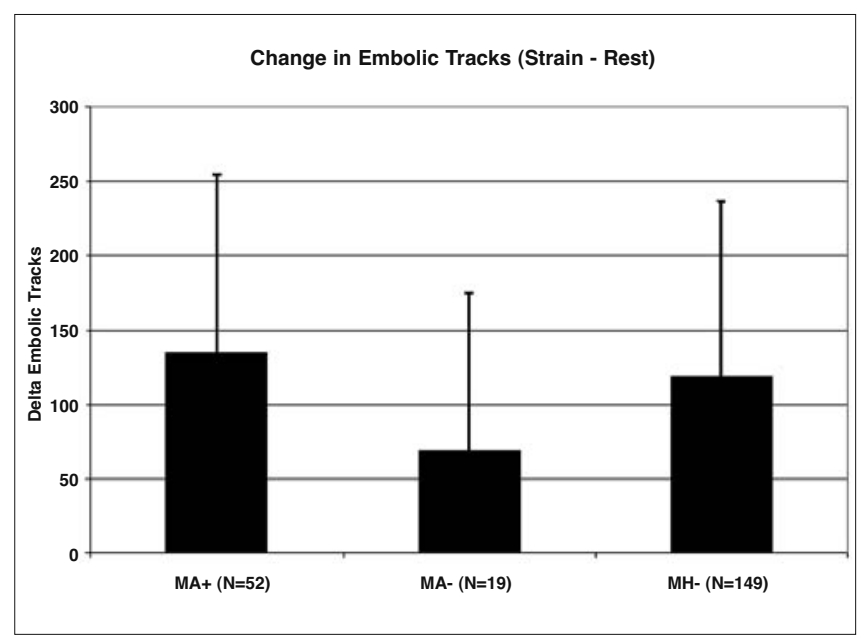

Fig. 3 Change in RLS from rest to strain in migraineurs with aura $(\mathrm{MA}+)$, migraineurs without aura (MA-) and non-migraineurs (MH-). The omnibus comparison was not significant $\left(\chi^{2}=4.9\right.$, $p=0.09)$; however, MA+ had a larger change in RLS from rest to strain than MA- $(Z=-2.2, p=0.03)$ mum and septum secundum on TEE, so their results are not directly comparable to balloon stretch diameter. Two population-based studies have reported that isolated ASA was an independent risk factor for cerebrovascular events [15, 16]. Meissner et al. [15] reported that risk of cerebrovascular events was greater in subjects with ASA (hazard ratio 3.72, 95\% CI 0.88-15.71). Di Tullio et al. [16] reported similar results for the Northern Manhattan Study (hazard ratio 3.66, 95\% CI 0.88-15.30). Both of these cohorts were older (mean age 66.9 and 68.7 years, respectively) and had a high percentage of hypertension ( $55 \%$ and $67 \%$, respectively), which makes it difficult to adjudicate the independent contribution of PFO to neurological events. In addition, the Northern Manhattan Study had a $22 \%$ incidence of diabetes mellitus [16] relative to $10 \%$ in the present study.

The finding that MA- had a higher degree of RLS at rest compared to MA+ was unexpected. In a previous study, migraineurs with and without aura had larger degrees of RLS measured by single-gated TCD than non-migraineurs [20]; however, the authors found no difference between migraine groups. Carod-Artal et al. [5] reported that MA+ had a higher incidence of PFO than migraineurs without aura (MA-) $(52 \%$ vs. $34 \%, p=0.02$ ), but, in contrast to our findings, found no difference in the degree of RLS between the two groups of migraineurs (shower or curtain pattern: MA+, 77\%; MA-, $64 \%, p=0.55$ ). Wilmshurst et al. [6] reported that a significantly higher proportion of MA+ with stroke had clinically relevant RLS as assessed by transthoracic echocardiography than $\mathrm{MA}+$ without stroke and historical normal controls ( $>6$ microbubbles; $84 \%, 38 \%$ and $12 \%$, respectively). The balloon diameter at PFO closure corresponded to the degree of RLS in this study; however, no correlation coefficient was reported [6]. In one study, migraineurs with aura were more likely to have headache triggers associated with Valsalva-provoking activities and have larger RLS than migraineurs without aura [30]. Spontaneous large RLS and MA+ were both risk factors for recurrent stroke in patients with PFO (large RLS OR 2.42, 95\% CI 1.66-5.04; MA+ OR 3.87, 95\% CI 1.75-8.50) [31]. Freedom from recurrent MA+ five years after PFO closure was $85 \%$ (95\% CI 57-97\%), and the recurrent stroke rate was $5.3 \%$ in another study [32]. However, degree of RLS was not associated with risk of stroke in a population-based case-control study of stroke and TIA [33]. The above findings are in contrast to the results of the current study, which demonstrated a non-significant trend in MA- to have a higher percentage of recurrent stroke prior to $\mathrm{PFO}$ closure.

Migraineurs with aura and PFO may have an increased risk for shunting of cellular and/or humoral substances from the venous circulation to the cerebral circulation during strain [8]. By evading pulmonary filtration, these substances may trigger migraines and increase the risk of stroke. The literature to support this hypothesis is controversial and inconsistent. Although non-randomised trials have shown reductions in 
migraines with aura after transcatheter closure of $\mathrm{PFO}$ [10-12], only $3 / 70(4.3 \%)$ of patients in the randomised Migraine Intervention with STARFlex Technology (MIST) trial experienced total cessation of migraines after PFO closure; $42 \%$ had a $>50 \%$ reduction in headache days relative to $23 \%$ of patients in the sham closure group $(p=0.038)$ [34]. The initial MIST data were not corrected for imbalances in baseline characteristics. In addition, the MIST cohort may have included a higher number of patients refractory to medical treatment than the non-randomised trials [9]. In response to the results of the MIST trial, the Council of the European Headache Federation advised that there was insufficient evidence to use PFO closure for migraine prophylaxis [35].

This study has several limitations. The design of the present study did not allow for age- and gender-matching of patient groups. The patient sample was relatively small (total $\mathrm{n}=220$ ) and self-selected (undergoing elective transcatheter PFO closure for prevention of recurrent neurovascular events), which make the results of this study difficult to generalise. The potential for selection bias exists because a probe patent PFO was a criterion for PFO closure, which may have assured a minimum size of lesion. At rest, 28 (54\%) MA+, 15 (79\%) MA- and 68 (46\%) MH- had large RLS; at strain, 51 (98\%) MA+, 19 (100\%) MA- and 133 (89\%) MH- had large RLS. In addition, 34\% of the cohort had coexisting ASA, which is higher than population-based studies (i.e., non-PFO closure)
$[16,36]$ but not necessarily higher than studies involving PFO closure [37, 38]. Utilisation of the Spencer grading system [23], with a maximum grade of $\mathrm{V}$ ( $>300$ embolic tracks), may have reduced the ability to detect differences after calibrated Valsalva between groups, as $92 \%$ of all patients had large RLS (Grades IV-V) following Valsalva. The study was slightly underpowered to detect a significant difference between the percentages of patients in each group who presented with large RLS. It is possible that some of the TIAs reported by patients were actually complex migraines without headache and we were unable to discern this distinction. Femoral vein injection of agitated saline may have yielded different results on the degree of RLS than antecubital vein injection, as blood flow from the inferior vena cava is directed at the right atrial septum [39].

In conclusion, significant differences were found in the proportion of large RLS at rest and at strain, between migraineurs and non-migraineurs who were referred for transcatheter PFO closure for prevention of recurrent cerebrovascular events. Atrial septal characteristics did not differ between groups. Therefore, it appears unlikely that migraine status can predict anatomical PFO characteristics. Further investigations of the degree of RLS and atrial septal characteristics are needed to confirm the relation between migraine and PFO, particularly the relation between large RLS and risk of recurrent neurological events.

\section{References}

1. Hu XH, Markson LE, Lipton RB et al (1999) Burden of migraine in the United States: disability and economic costs. Arch Intern Med 159:813-818

2. Gerth WC, Carides GW, Dasbach EJ et al (2001) The multinational impact of migraine symptoms on healthcare utilisation and work loss.

Pharmacoeconomics 19:197-206

3. Etminan M, Takkouche B, Isorna FC, Samii A (2005) Risk of ischaemic stroke in people with migraine: systematic review and meta-analysis of observational studies.

Br Med J 330:63-66

4. Del Sette M, Angeli S, Leandri M et al (1998) Migraine with aura and rightto-left shunt on transcranial Doppler: a case-control study. Cerebrovasc Dis 8:327-330
5. Carod-Artal FJ, da Silveira Ribeiro L, Braga $\mathrm{H}$ et al (2006) Prevalence of patent foramen ovale in migraine patients with and without aura compared with stroke patients. A transcranial Doppler study. Cephalalgia 26:934-939

6. Wilmshurst P, Nightingale S, Pearson $M$ et al (2006) Relation of atrial shunts to migraine in patients with ischemic stroke and peripheral emboli. Am J Cardiol 98:831-833

7. Wilmshurst P, Nightingale S (20001) Relationship between migraine and cardiac and pulmonary right-to-left shunts. Clin Sci (Lond) 100:215-220

8. Post MC, Budts W (2006) The relationship between migraine and rightto-left shunt: Fact or fiction? Chest 130:896-901
9. Schwerzmann M, Nedeltchev K, Meier B (2007) Patent foramen ovale closure: A new therapy for migraine. Catheter Cardiovasc Interv 69:277-284

10. Morandi E, Anzola GP, Angeli S et al (2003) Transcatheter closure of patent foramen ovale: a new migraine treatment? J Interv Cardiol 16:39-42

11. Azarbal B, Tobis J, Suh W et al (2005) Association of interatrial shunts and migraine headaches: impact of transcatheter closure. J Am Coll Cardiol 45:489-492

12. Reisman M, Christofferson RD, Jesurum J et al (2005) Migraine headache relief after transcatheter closure of patent foramen ovale. J Am Coll Cardiol 45:493-495 
13. Hagen PT, Scholz DG, Edwards WD (1984) Incidence and size of patent foramen ovale during the first 10 decades of life: an autopsy study of 965 normal hearts. Mayo Clin Proc 59:17-20

14. Cabanes L, Mas JL, Cohen A et al (1993) Atrial septal aneurysm and patent foramen ovale as risk factors for cryptogenic stroke in patients less than 55 years of age. A study using transesophageal echocardiography. Stroke 24:1865-1873

15. Meissner I, Khandheria BK, Heit JA et al (2006) Patent foramen ovale: innocent or guilty? Evidence from a prospective population-based study. J Am Coll Cardiol 47:440-445

16. Di Tullio MR, Sacco RL, Sciacca RR et al (2007) Patent foramen ovale and the risk of ischemic stroke in a multiethnic population. J Am Coll Cardiol 49:797-802

17. Carerj S, Narbone MC, Zito C et al (2003) Prevalence of atrial septal aneurysm in patients with migraine: an echocardiographic study. Headache 43:725-728

18. Schuchlenz HW, Weihs W, Horner S, Quehenberger F (2000) The association between the diameter of a patent foramen ovale and the risk of embolic cerebrovascular events. Am J Med 109:456-462

19. Schwerzmann M, Nedeltchev K, Lagger F et al (2005) Prevalence and size of directly detected patent foramen ovale in migraine with aura. Neurology 65:1415-1418

20. Anzola GP, Morandi E, Casilli F, Onorato E (2006) Different degrees of right-to-left shunting predict migraine and stroke: data from 420 patients. Neurology 66:765-767

21. Anzola GP, Magoni M, Guindani M et al (1999) Potential source of cerebral embolism in migraine with aura: a transcranial Doppler study. Neurology 52:1622-1625

22. The International Classification of Headache Disorders: $2^{\text {nd }}$ edition. Cephalalgia 2004: 24 Suppl 1:9-160
23. Spencer MP, Moehring MA, Jesurum J et al (2004) Power m-mode transcranial Doppler for diagnosis of patent foramen ovale and assessing transcatheter closure. J Neuroimaging 2004:14:342-349

24. Zanchetta M, Rigatelli G, Onorato E (2003) Intracardiac echocardiography and transcranial Doppler ultrasound to guide closure of patent foramen ovale. J Invasive Cardiol 15:93-96

25. Hanley PC, Tajik AJ, Hynes JK et al (1985) Diagnosis and classification of atrial septal aneurysm by two-dimensional echocardiography: report of 80 consecutive cases. J Am Coll Cardiol 6:1370-1382

26. Post MC, Thijs V, Herroelen L, Budts WI (2004) Closure of a patent foramen ovale is associated with a decrease in prevalence of migraine. Neurology 62:1439-1440

27. Gori S, Morelli N, Fanucchi S et al (2006) The extent of right-to-left shunt fails to correlate with severity of clinical picture in migraine with aura. Neurol Sci 27:14-17

28. Hijazi Z, Wang Z, Cao Q et al (2001) Transcatheter closure of atrial septal defects and patent foramen ovale under intracardiac echocardiographic guidance: feasibility and comparison with transesophageal echocardiography. Catheter Cardiovasc Interv 52:194-199

29. Lamy C, Giannesini C, Zuber M et al (2002) Clinical and imaging findings in cryptogenic stroke patients with and without patent foramen ovale: the PFO-ASA Study. Atrial Septal Aneurysm. Stroke 33:706-711

30. Tembl J, Lago A, Sevilla T et al (2007) Migraine, patent foramen ovale and migraine triggers. J Headache Pain 8:7-12

31. Giardini A, Donti A, Formigari R et al (2006) Spontaneous large right-to-left shunt and migraine headache with aura are risk factors for recurrent stroke in patients with a patent foramen ovale. Int J Cardiol 120:357-362
32. Giardini A, Donti A, Formigari R et al (2006) Long-term efficacy of transcatheter patent foramen ovale closure on migraine headache with aura and recurrent stroke. Catheter Cardiovasc Interv 67:625-629

33. Petty GW, Khandheria BK, Meissner I et al (2006) Population-based study of the relationship between patent foramen ovale and cerebrovascular ischemic events. Mayo Clin Proc 81:602-608

34. Dowson AJ, Wilmshurst P, Muir KW et al (2006) A prospective, multicenter, randomized, double blind, placebocontrolled trial to evaluate the efficacy of patent foramen ovale closure with the STARFlex septal repair implant to prevent refractory migraine headaches: the MIST Trial. Neurology 67:185

35. Diener HC (2007) Patent foramen ovale and migraine: no reason to intervene. J Headache Pain 8:3-6

36. Meissner I, Whisnant JP, Khandheria BK (1999) Prevalence of potential risk factors for stroke assessed by transesophageal echocardiography and carotid ultrasonography: the SPARC study. Stroke Prevention: Assessment of Risk in a Community. Mayo Clin Proc 74:862-869.

37. Windecker S, Wahl A, Nedeltchev K et al (2004) Comparison of medical treatment with percutaneous closure of patent foramen ovale in patients with cryptogenic stroke. J Am Coll Cardiol 44:750-758

38. Schuchlenz HW, Weihs W, Berghold A et al (2005) Secondary prevention after cryptogenic cerebrovascular events in patients with patent foramen ovale. Int J Cardiol 101:77-82

39. Hamann GF, Schatzer-Klotz D, Frohlig $\mathrm{G}$ et al (1998) Femoral injection of echo contrast medium may increase the sensitivity of testing for a patent foramen ovale. Neurology 50:1423-1428 